\title{
The task of direct piezoeffect for a bi-morth plate
}

\author{
Dmitriy Shlyahin ${ }^{1, *}$, and Olesya Ratmanova ${ }^{1}$ \\ ${ }^{1}$ Samara State Technical University, Academy of Architecture and Civil Engineering, \\ Molodogvardeyskaya St., 194, Samara, 443001, Russia
}

\begin{abstract}
The research focuses on the dynamic axisymmetric task for a round bi-morph structure consisting of a metal support plate and a piezoceramic axially polarized plate. Its bending oscillations are carried out because of the actions of mechanical load (normal stresses) on its end surface, which is an arbitrary time and radial coordinate function. The rigid and hinged support of the plate cylindrical surface is taken into account. The value of the induced field is calculated by determining the potential on the metal support plate. To solve the task of the theory of the elasticity in a three-dimensional model, the authors apply the Hankel finite integral transformations along the axial coordinate and generalized transformation along the radial variable. Besides, at each stage of the study the standardization procedure is carried out. In the first case it is connected with the use of mixed boundary conditions along the radial coordinate to the mixed form, and in the second case heterogeneous boundary conditions are presented as homogenious. The obtained calculation ratios make it possible to determine the optimal thickness of the piezoceramic plate, which allows to make the most effective transformation of the external mechanical effect into an electrical signal. In addition, it is possible to determine changing characteristics of electromagnetic field according to the height of the piezoceramic plate. This principle can be used in the calculation and design of bi-morph systems with graded-varying thickness and rigidity.
\end{abstract}

\section{Introduction}

The calculation of piezoceramic multilayer structures, in the main one, is carried out by means of applied theories for thin plates [1,2] in which kinematic hypotheses are supplemented by similar assumptions about the changing characteristics of electromagnetic field [3-9]. To take into account physical fields connection in multilayer piezoceramic plates, the authors of papers $[10,11]$ conducted more extensive research and worked out a closed solution of non-stationary problem of inverse piezoeffect in a three-dimentional model.

\footnotetext{
* Corresponding author: d-612-mit2009@yandex.ru
} 


\section{Materials and methods}

Let a round two-layer plate occupying the region in a cylindrical coordinate $\left(r_{*}, \theta, z_{*}\right)$ occupies region $\Omega\left\{0 \leq r_{*} b, 0 \leq \theta<2 \pi, 0 \leq z_{*} \leq h^{*}\right\}$ It consists of a piezoceramic element of $h_{1}^{*}$ height, made of a material with a hexagonal crystalline lattice of $6 \mathrm{~mm}$ Class, and a metal support plate $h_{2}^{*}$ with thickness $\left(h^{*}=h_{1}^{*}+h_{2}^{*}\right)$. Axisymmetric bending oscillations are aroused by the action of mechanical dynamic load (normal stresses) $q^{*}\left(r_{*}, t_{*}\right)$, which is an arbitrary function of radial coordinate $r_{*}$ and time. Electrodes connection to the measuring instrument allows to fix the amount and mode of electrical voltage $V^{*}\left(t_{*}\right)$. We consider cases of rigid and hinged support of a cylindrical surface of the structure under analysis.

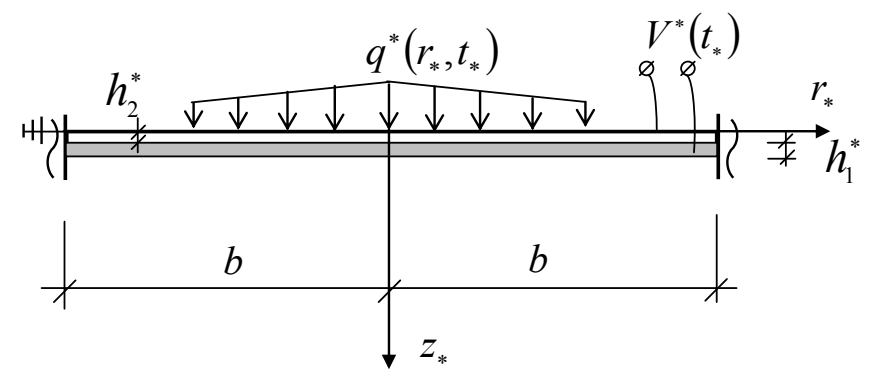

Fig. 1. Bi-morph plate.

Differential equations of motion and electrostatics, as well as initial boundary conditions in a cylindrical coordinates system and dimensionless form, are as follows:

$$
\begin{aligned}
& \frac{\partial}{\partial r} \nabla U+\frac{C_{55}^{(s)}}{C_{11}^{(s)}} \frac{\partial^{2} U}{\partial z^{2}}+\frac{\left(C_{13}^{(s)}+C_{55}^{(s)}\right)}{C_{11}^{(s)}} \frac{\partial^{2} W}{\partial r \partial z}+\frac{\left(e_{31}+e_{15}\right)}{e_{33}} \frac{\partial^{2} \phi}{\partial r \partial z}-\Phi^{(s)} \frac{\partial^{2} U}{\partial t^{2}}=0, \\
& \frac{C_{55}^{(s)}}{C_{11}^{(s)}} \nabla \frac{\partial W}{\partial r}+\frac{C_{33}^{(s)}}{C_{11}^{(s)}} \frac{\partial^{2} W}{\partial z^{2}}+\frac{\left(C_{13}^{(s)}+C_{55}^{(s)}\right)}{C_{11}^{(s)}} \frac{\partial}{\partial z} \nabla U+\frac{e_{15}}{e_{33}} \nabla \frac{\partial \phi}{\partial r}+\frac{\partial^{2} \phi}{\partial z^{2}}-\Phi^{(s)} \frac{\partial^{2} W}{\partial t^{2}}=0, \\
& \frac{\left(e_{31}+e_{15}\right)}{e_{33}} \frac{\partial}{\partial z} \nabla U+\frac{e_{15}}{e_{33}} \nabla \frac{\partial W}{\partial r}+\frac{\partial^{2} W}{\partial z^{2}}-\frac{C_{11}^{(l)} \varepsilon}{e_{33}^{2}} \nabla \frac{\partial \phi}{\partial r}-\frac{C_{11}^{(l)} \varepsilon_{11}}{e_{33}^{2}} \frac{\partial^{2} \phi}{\partial z^{2}}=0 ;
\end{aligned}
$$

$r=0,1$

$$
W(0, z, t)<\infty, \quad U(0, z, t)<\infty, \quad \phi(0, z, t)<\infty,
$$

$$
D_{r \mid r=1}=-\frac{C_{11}^{(l)} \varepsilon_{11}}{e_{33}^{2}} \frac{\partial \phi}{\partial r}+\frac{e_{15}}{e_{33}}\left(\frac{\partial W}{\partial r}+\frac{\partial U}{\partial z}\right)=0
$$

- rigid support: $\quad W(1, z, t)=0, \quad U(1, z, t)=0$

- hinge support: $\quad W(1, z, t)=0$

$z=0$

$$
\begin{gathered}
\sigma_{r r \mid r=1}=\frac{C_{11}^{(s)}}{C_{11}^{(l)}} \frac{\partial U}{\partial r}+\frac{C_{12}^{(s)}}{C_{11}^{(l)}} \frac{U}{r}+\frac{C_{13}^{(s)}}{C_{11}^{(l)}} \frac{\partial W}{\partial z}+\frac{e_{31}}{e_{33}} \frac{\partial \phi}{\partial z}=0 ; \\
\sigma_{z z}=\frac{C_{13}^{(s)}}{C_{11}^{(l)}} \nabla U+\frac{C_{13}^{(s)}}{C_{11}^{(l)}} \frac{\partial W}{\partial z}=q ; \quad \sigma_{r z}=\frac{C_{55}^{(s)}}{C_{11}^{(l)}}\left(\frac{\partial W}{\partial r}+\frac{\partial U}{\partial z}\right)=0 ;
\end{gathered}
$$




$$
\begin{aligned}
& z=h_{2} \quad \frac{C_{13}^{(1)}-C_{13}^{(2)}}{C_{11}^{(1)}} \nabla U+\frac{C_{33}^{(1)}-C_{33}^{(2)}}{C_{11}^{(l)}} \frac{\partial W}{\partial z}+\frac{\partial \phi}{\partial z}=0, \\
& \frac{C_{55}^{(1)}-C_{55}^{(2)}}{C_{11}^{(1)}}\left(\frac{\partial W}{\partial r}+\frac{\partial U}{\partial z}\right)+\frac{e_{15}}{e_{33}} \frac{\partial \phi}{\partial r}=0, \quad \phi=0, \\
& U(z+0) U(z-0)=0, \quad W(z+0)-W(z-0)=0 ; \\
& z=h \quad \sigma_{z z}=\frac{C_{13}^{(l)}}{C_{11}^{(l)}} \nabla U+\frac{C_{13}^{(1)}}{C_{11}^{(l)}} \frac{\partial W}{\partial z}+\frac{\partial \phi}{\partial z}=0 ; \\
& \sigma_{r z}=\frac{C_{55}^{(l)}}{C_{11}^{(l)}}\left(\frac{\partial W}{\partial r}+\frac{\partial U}{\partial z}\right)+\frac{e_{15}}{e_{33}} \frac{\partial \phi}{\partial r}=0, \quad D_{z}=-\frac{C_{11}^{(l)} \varepsilon_{33}}{e_{33}^{2}} \frac{\partial \phi}{\partial z}+\frac{e_{31}}{e_{33}} \nabla U+\frac{\partial W}{\partial z}=0 ; \\
& t=0 \\
& U(r, z, 0)=U_{0}(r, z), \quad \dot{U}(r, z, 0)=\dot{U}_{0}(r, z), \\
& W(r, z, 0)=W_{0}(r, z), \quad \dot{W}(r, z, 0)=\dot{W}_{0}(r, z) ;
\end{aligned}
$$

Here $\quad\left\{r, z, h, h_{1}, h_{2}\right\}=\left\{r_{*}, z_{*}, h^{*}, h_{1}{ }^{*}, h_{2}{ }^{*}\right\} / b ; t=t_{*} b^{-1} \sqrt{C_{11}^{(2)} / \rho^{(2)}} ; \quad U\left(r_{*}, z_{*}, t_{*}\right)$, $W\left(r_{*}, z_{*}, t_{*}\right), \sigma_{p m}\left(r_{*}, z_{*}, t_{*}\right)$ - are components of the movement vector and the stress tensor $(p, m=r, z) ; D_{r}\left(r_{*}, z_{*}, t_{*}\right), D_{z}\left(r_{*}, z_{*}, t_{*}\right), \phi^{*}\left(r_{*}, z_{*}, t_{*}\right)$ - are components of the induction vector and the electric field potential; $e_{m k}, \varepsilon_{11}, \varepsilon_{33}$ - are piezomodules and coefficients of dielectric permeability of the elastic material; $(m, k=\overline{1,5}) ; \rho^{(s)}, C_{m k}{ }^{(s)}-$ are the spatial density and elasticity modules of piezoceramic $(s=1)$ and elastic $(s=1)$ materials; $U_{0}, \dot{U}_{0}, W_{0}, W_{0}-$ are displacements and speed of movement $q=\frac{q}{c_{11}^{(1)}}, \Phi^{(1)}=$ $\frac{C_{11}^{(2)} \rho^{(1)}}{C_{11}^{(1)} \rho^{(2)}}, \Phi^{(2)}=1$, known at the initial time. $\nabla=\frac{\partial}{\partial r}+\frac{1}{r}$

\section{Results}

At the first stage of the solution the boundary problem (1) - (8) is solved by the method of the Hankel finite integral transformations according to the radial coordinate $r$. Here, the transformation can only satisfy the mixed boundary conditions.

To fulfill this requirement we need:

- for rigid support: the first equality (3) should be replaced by the condition of shearing stresses unavailability on cylindrical surfaces of plate $(r=1)$ :

$$
\sigma_{r z \mid r=k}=\frac{C_{55}^{(s)}}{C_{11}^{(l)}}\left(\frac{\partial W}{\partial r}+\frac{\partial U}{\partial z}\right)+\frac{e_{15}}{e_{33}} \frac{\partial \phi}{\partial r}=0
$$

- for hinged support: the last ratio (2) should be replaced by the condition of availability of the electric field potential $\phi_{1}(z, t)$ on cylindrical surfaces at : 


$$
\phi(1, z, t)=0
$$

As a result, we get a new boundary task regarding functions $u, \omega, \varphi$. Here, differential equations (1), the first condition (5), the first three conditions (6) and the equalities (7) become heterogeneous, and boundary conditions (2) - (4) take the following form:

- rigid fixing: $\quad U(1, z, t)=, \frac{\partial W}{\partial r_{\mid z=1}}=0, \frac{\partial \phi}{\partial r_{\mid z=1}}=0$

-hinge support: $W=(1, z, t)=0, \quad \phi(1, z, t)=0, \quad \nabla U_{\mid r=1}=0$

Then we sequentially apply finite integral transformations by coordinates $r$ and $z$ to the boundary problem (1) - (8), (9), (10) relative to the functions $u, \omega, \varphi$. The final expressions of functions $U(r, z, t), W(r, z, t), \phi(r, z, t)$ are obtained while sequentially applying reciprocation formula to the transformants.

\section{Conclusions}

The study examines a bi-morph plate with the following physical and geometrical characteristics of axial polarized piezoceramic plates of TSTC-19 structure and metal steel substrate under action of evenly distributed load of intensity $q_{0}$ and $\theta$ frequency $(\theta=$ $\lambda_{11} / 5, \lambda_{11}$ - being the first natural oscillation frequency):

$$
q(r, t)=q(t)=q_{0} \sin (\theta t)
$$

Numerical analysis of results allows to make the following conclusions:

1) It is possible to determine the optimal height of a piezoceramic plate, which allows to effectively transform external mechanical effect into an induce electric signal for a structure with a specified thickness of substrate.

2) Amplitude values of electrical impulse $V(t)$ at hinged support of a plate are considerably more intensive, than in case of its contour rigid fixing.

\section{References}

1. V.M. Alexandrov, M.I. Chebakov, Analytical methods in contact problems of elasticity theory (Moscow, 2004)

2. S.M. Aizikov, A.V. Belokon, V.M. Alexandrov, L.I. Krenev, I.S. Trubchik, Contact problems of elasticity theory for heterogeneous media (Moscow, 2006)

3. V. Tsaplev, R. Konovalov, K. Abbakumov, J. of Power and Energy Eng., 3, 63-68 (2015)

4. J.E. Jam, M. Khosravi, N. Namdaran, Metall, Mater. Eng. 19(1), 45-63 (2013)

5. V.L. Karlash, Resonance Electro-Mechanic Vibration of Piezo-Ceramic Plates, Int. Appl. Mech.,7, 535-541 (2005)

6. S.A. Vatulyan, A.A. Rynkova, News of Russian Academy of Sciences. MTT., 4, $114-$ 122 (2007)

7. Y. Wang, R.Q. Xu, H.J. Ding, Acta Mechanica, 215, 287-305 (2010) 
8. D.A. Shlyahin, News of Ussian Academy of Sciences, MTT, 2, 77-85 (2013)

9. D.A. Shlyakhin, O.V. Kazakova, Procedia Engineering, 91, 69-74 (2014)

10. Yu.E. Senitskiy, News of Saratov university, Series: Mat., Mech., Inform, 11, 3 Part 1. 2011)

11. Yu.E. Senitskiy, Actual problems of mathematics and mechanics, 133-134 (2013) 